\title{
Effects of IL-6 on amylase secretion and calcium signalling in pancreatic AR42J cells: modulation by membrane fatty acid composition
}

\author{
N. Audi $^{1}$, M. A. Martínez ${ }^{1}$, M. D. Mesa ${ }^{2}$, E. Martínez-Victoria ${ }^{1}$, M. Mañas ${ }^{1}$ and M. D. Yago ${ }^{1}$ \\ ${ }^{1}$ Institute of Nutrition and Food Technology, Departments of Physiology and ${ }^{2}$ Biochemistry and Molecular Biology, \\ University of Granada, Spain
}

Oleic acid is a typical component of the Mediterranean diet. The type of dietary fat strongly influences the fatty acid (FA) composition of rat pancreatic cell membranes, and this effect is associated with changes in the functionality of viable pancreatic acini ${ }^{(1,2)}$. The AR42J cell line is a useful tool for assessing the effect of membrane compositional changes on acinar cell function ${ }^{(3)}$. The aim was to study the effects of chronic treatment with IL-6 (400 pm for $48 \mathrm{~h}$ ) on amylase secretion and $\mathrm{Ca}^{2+}$ homeostasis in AR42J cells, and to establish whether these effects are influenced by different membrane FA composition.

The membrane FA composition of AR42J cells was modified by growing them in medium enriched with oleic or linoleic acid, as described previously ${ }^{(3)}$. Amylase activity was determined and expressed as a percentage of the initial total amylase content that was released into the extracellular medium during incubation. $\mathrm{Ca}^{2+}$ mobilization (expressed as F340:F380) was determined by epifluorescence microscopy. Cells were loaded with the fluorescent ratiometric $\mathrm{Ca}^{2+}$ indicator fura- 2 . For quantification of fluorescence, cells were alternately excited at 340 and $380 \mathrm{~nm}$ using a high-speed monochromator. ANOVA was performed to compare amylase secretion between groups treated with or without IL-6. The $\mathrm{Ca}^{2+}$ decay constant for each group was calculated and mean values were compared by ANOVA.

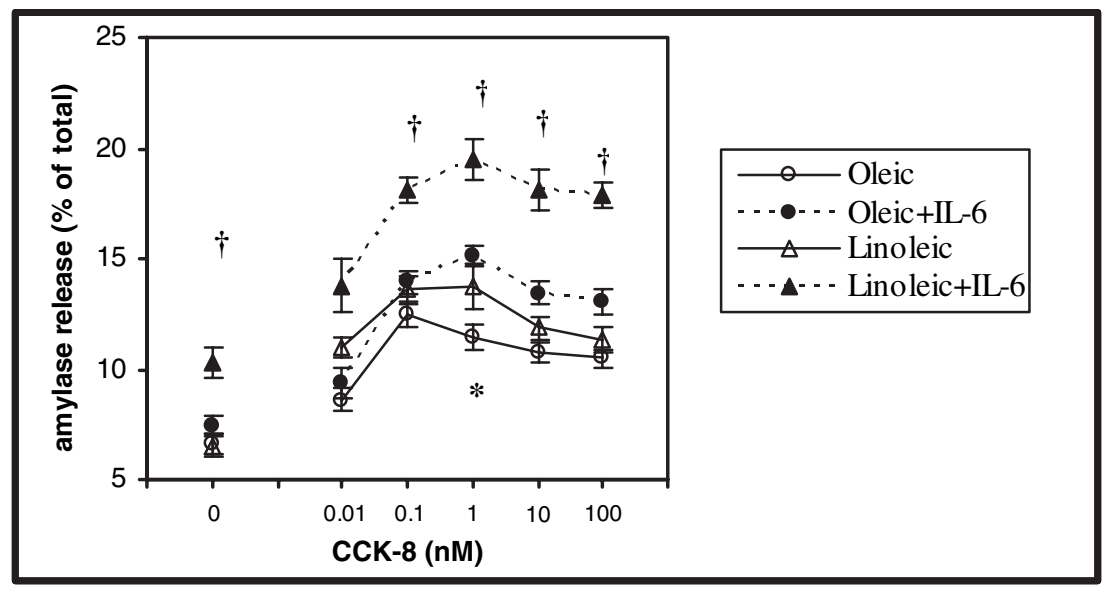

Figure. Amylase release ( $\%$ total content) evoked by different concentrations of cholecystokinin octapeptide (CCK-8). Values are means with their standard errors represented by vertical bars ( $n 16$ for all groups). Mean value for the oleic acid group was significantly different from that for the oleic + IL-6 group: $* P<0.05$. Mean values for the linoleic acid group were significantly different from those for the linoleic acid + IL-6 group: $\dagger P<0.05$.

Table. $\mathrm{Ca}^{2+}$ response evoked by perfusion with CCK-8 $(1 \mathrm{~nm})$ in AR42J cells with different membrane FA profiles treated for $48 \mathrm{~h}$ with IL-6 or vehicle

\begin{tabular}{lccc}
\hline & \multicolumn{2}{c}{ Peaks (340:380 intensity) } & \multicolumn{2}{c}{$\mathrm{Ca}^{2+}$ decay constants (/s) } & Mean \\
\cline { 2 - 4 } & Mean & SE & $0.0034^{*}$ \\
Oleic acid $(n$ 44) & 2.2070 & 0.0861 & 0.0028 \\
Oleic acid + IL-6 $(n$ 39) & 2.1297 & 0.1037 & 0.0002 \\
Linoleic acid $(n$ 42) & 2.1930 & 0.1112 & 0.0002 \\
Linoleic acid + IL-6 $(n$ 29) & 2.1457 & 0.0978 & 0.0003 \\
\hline
\end{tabular}

$n$, No. of cells measured in each group. Mean value for the oleic acid group was significantly different from that for the oleic + IL- 6 group: $* P<0.05$.

Membranes rich in oleic acid were not affected by the action of IL-6 at different concentrations of CCK-8, while membranes rich in linoleic acid were more sensitive to the effects of IL-6 in relation to basal and stimulated amylase secretion. Dietary fat and IL-6 did not affect $\mathrm{Ca}^{2+}$ peaks elicited by 1 nM-CCK-8. It would be useful to know whether the consumption of olive oil (rich in oleic acid) can prevent and/or attenuate the effects of pancreatic inflammatory processes compared with sunflower oil (rich in linoleic acid).

1. Martinez MA, Lajas AI, Yago MD et al. (2004) Nutrition 20, 536-541.

2. Yago MD, Diaz RJ, Ramirez R, Martinez MA, Mañas M \& Martinez-Victoria E (2004) Br J Nutr 9, $1227-1234$.

3. Audi N, Mesa MD, Martinez MA, Martinez-Victoria E, Mañas M \& Yago MD (2007) Exp Biol Med $232,532-541$. 